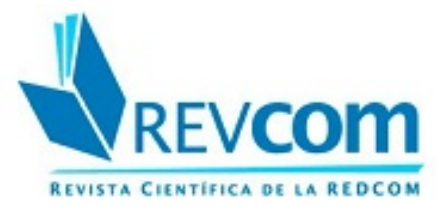

REVCOM. Revista científica de la red de carreras de Comunicación Social

ISSN: 2451-7836

redcom.revcom@gmail.com

Universidad Nacional de La Plata

Argentina

\title{
Investigar la realidad para transformarla
}

Aguerre, Natalia

Investigar la realidad para transformarla

REVCOM. Revista científica de la red de carreras de Comunicación Social, núm. 10, 2020

Universidad Nacional de La Plata, Argentina

DOI: https://doi.org/10.24215/24517836e034 
Artículos

\title{
Investigar la realidad para transformarla
}

\author{
Investigar a realidade para transformar \\ Investigating Reality to Transform it \\ Natalia Aguerre \\ Facultad de Periodismo y Comunicación Social. \\ Universidad Nacional de La Plata, Argentina \\ aguerre.natalia@yahoo.com
}

DOI: https://doi.org/10.24215/24517836e034

Recepción: 14 Febrero 2020

Aprobación: 10 Junio 2020

\section{Resumen:}

El artículo propone reflexionar sobre los retos que enfrentan los investigadores en comunicación en la tarea de producir y difundir saberes. El mismo surgió a partir de los debates llevados adelante por un equipo de la Facultad de Periodismo y Comunicación Social de la Universidad de La Plata, que integra el proyecto: Estrategias de comunicación de las organizaciones sociales para la incidencia y la participación en las políticas públicas. Lo público, redes sociales, Estado y ciudadanía. En virtud de este marco, se propone exponer las maneras en que el investigador en comunicación articula los aportes científicos con las acciones de las organizaciones sociales que tienen como objetivo la participación e incidencia para la transformación social.

Palabras Clave: Comunicación, Investigación, Participación, Organizaciones sociales.

\section{Resumo:}

O artigo propõe refletir sobre os desafios que os pesquisadores em comunicação enfrentam na tarefa de produzir e disseminar conhecimento. O mesmo surgiu dos debates realizados por uma equipe da Faculdade de Jornalismo e Comunicação Social da Universidade de La Plata, que integra o projeto: Estratégias de comunicação das organizações sociais para a defesa e participação na política pública. . O público, redes sociais, Estado e cidadania. Sob este referencial, propõe-se apresentar as formas pelas quais o pesquisador em comunicação articula as contribuições científicas com as ações das organizações sociais que têm como objetivo a participação e incidência para a transformação social.

Palavras-ChaVE: Comunicação, Investigação, Participação, Organizações Sociais.

\section{Abstract:}

The article proposes to reflect on the challenges that researchers in communication face in the task of producing and disseminating knowledge. The same arose from the debates carried out by a team of the Faculty of Journalism and Social Communication of the University of La Plata, which integrates the project: Communication strategies of social organizations for advocacy and participation in public policy. The public, social networks, State and citizenship. Under this framework, it is proposed to present the ways in which the researcher in communication articulates the scientific contributions with the actions of the social organizations that have as objective the participation and incidence for the social transformation.

KEYWORDS: Communication, Investigation, Participation, Social Organizations.

\section{INTRODUCCIÓN}

En los últimos tiempos, el avance de la investigación científica en Latinoamérica ha hecho que surjan nuevos retos para los investigadores en su relación con la innovación tecnológica, el desarrollo y la producción de saberes e instancias para el bienestar social. Desde el campo de la comunicación se hace necesario interrogarnos acerca de los modos de crear y difundir conocimiento y de cómo con ellos intervenimos en la esfera pública para promover cambios sociales. Pensar el trabajo del investigador en comunicación implica poner en tensión crítica la generación de significados, los cuales se van desarrollando en lucha por los sentidos políticos/culturales que circulan en el entramado social. Pero también se requiere reflexionar sobre 
las propias prácticas de la labor investigativa; es decir, sobre las maneras en que se aborda la realidad mediante la vinculación permanente entre la teoría científica y la praxis.

El propósito de este artículo es reflexionar sobre el rol del investigador en comunicación presentando algunos aspectos del proceso de planificación estratégica comunicacional con organizaciones sociales en este caso, InterRedes-, en el marco del proyecto de investigación "Estrategias de comunicación de las organizaciones sociales para la incidencia y la participación en las políticas públicas. Lo público, redes sociales, Estado y ciudadanía" inscripto en la Facultad de Periodismo y Comunicación Social (FPyCS) de la Universidad Nacional de La Plata (UNLP)i.

\section{CONOCER/HACER}

Por ser una disciplina dinámica que intenta explorar y explicar las formas de producción de mensajes e intercambio de sentidos en los diferentes ámbitos y niveles del quehacer de los sujetos, la pregunta sobre desde qué aspectos analizarlos sigue siendo recurrente en nuestro campo.

La formulación de preguntas enmarcadas en la actividad investigativa demanda una base epistemológica que guíe al investigador en el planteamiento de las hipótesis y los métodos, en el contexto de un conjunto de teorías. En los años '60, la alianza entre la semiología y el paradigma de lo informacional pareció ser el modelo apropiado para interpretar los objetos de estudio. A medida que este método se aplicaba a fenómenos u acontecimientos particulares, se comenzó a percibir que existían variables que, siendo fundamentales para la comprensión holística de los mismos, quedaban al margen de los análisis establecidos. Algunos investigadores pusieron en crisis el canon semiológico-informativo expresando la necesidad de desplazar la observación de la comunicación como transmisión de la información hacia los procesos; o sea, hacia los actores, espacios, representaciones y/o demandas que se vinculan con y en las prácticas que se verifican en la sociedad. Esto significó considerar la comunicación desde los problemas y operaciones del intercambio social, desde las matrices de identidad y de los conflictos que configuran la cultura. Cultura que en América Latina implica inapelablemente el abordaje del rol del Estado/nación para comprender que, con la llegada de la modernidad, el concepto de ciudadanía en nuestros pueblos se organizó bajo un sujeto perteneciente a un territorio jurídico (Aguerre, 2017, s/p).

Como investigadores de la comunicación estamos ante una coyuntura que desafía nuestro conocimiento, los interrogantes formulados, y el motivo de la tarea de visibilizar y examinar las problemáticas sociales. Es por ello que resulta pertinente que el investigador indague las prácticas sociales -entendidas como "la acción colectiva, organizado o no, consciente o no, en la que intervienen actores diversos poniendo en juego sus saberes, intereses y poderes generando formas de interacción que adquieren significación y que provocan consecuencias sociales" (Uranga, 2014: 6), desde la perspectiva ciudadana donde el científico sea parte integral de lo que examina para vislumbrar la complejidad política/cultural, no solo de aquello que evalúa sino de las formas de crear conocimiento para poder construir miradas que transformen las maneras de relacionar el trabajo académico con dichas acciones colectivas.

Asumir la realización de estudios como un proceso en el cual los actores comprometidos participen activamente en la producción de información, análisis, planificación, y/o ejecución de acciones, permite que el conocimiento pueda llevarse a la esfera de la práctica de forma simultánea para concebir avances o cambios sociales. Añadimos, con las posturas de Paulo Freire (2005), que los sujetos cuando intentan comprender sus propias realidades logran una identificación con la labor de creación de saberes específicos que pueden utilizarse como instrumento para su empoderamiento. En esta línea, los miembros de las comunidades no se reducen a simples receptores, destinatarios o beneficiarios, sino que se los reconoce como depositarios y potenciales productores de conocimiento. Con lo cual, el trabajo del investigador en comunicación no radica en ser tan solo un experto, más bien en un facilitador o dinamizador que promueve un tipo estudios para ser utilizado en beneficio de la comunidad. Esto, en virtud de comprender la comunicación como 
la compleja trama de estrategias, medios y productos desarrollados en escenarios donde se dirime la lucha política dentro de la cual, los actores buscan desarrollar acciones que aporten a sus propósitos posicionándose como protagonistas de la lucha por el poder (Uranga, 2014: 3)

En función de la cita, la comunicación se encuentra atravesada por la acción política en términos de ciudadanía; en otras palabras, desde la relación entre el individuo y una comunidad, "en virtud de la cual el individuo es miembro de pleno derecho de esa comunidad y le debe lealtad permanente" (Cortina, 2001, como se citó en Uranga, 2014, p. 3).

Desde este enfoque, compartimos con Carol Weiss (1979), que el proceso de investigación debe considerar la edificación de puentes para la promoción de políticas públicas. A partir de esta premisa, Landry, Amara y Lamari (2001, p.347) argumentan que el modelo de interacción,

ocurre sobre la base de objetivos que se formulan a partir de las necesidades y los intereses de todos los actores del proceso, tanto de los investigadores como de los interesados e, inclusive, a través la participación de otros posibles actores. Este modelo se apoya fuertemente en los marcos de análisis focalizados en las diferencias culturales entre academia y política y en los atributos y características de la relación entre usuarios y productores. Se asume que, a mayor interacción entre los actores, mayor es la probabilidad de utilización del conocimiento

Observar al conjunto involucrado no como sujetos intervenidos sino como ciudadanos y portadores de conocimiento -al igual que el investigador-, facilita que los saberes y acciones que se establezcan en dichos vínculos puedan ser esgrimidos para la transformación de los entramados de poder que han contribuido a sostener desigualdades sociales. Debido a ello, la participación, en tanto capacidad constituyente de nuestra humanidad y por tanto un derecho fundamental (Aguerre, 2017), se vuelve clave para el análisis y la proyección de prácticas, dado que la misma al posibilitar el encuentro sociocultural de las personas configura "las tramas de relaciones comunicacionales conformando la densidad de la cultura" (Uranga y Thompson, 2016, p. 38). Los actores implicados ya no son objetos sino protagonistas en la detección de problemas para la toma de decisiones orientadas al cambio social, lo que viabiliza que el conocimiento (https://www.mono grafias.com/trabajos/epistemologia2/epistemologia2.shtml) se genere progresivamente en la participación. Así, los sujetos comprometidos toman posición con su palabra proporcionando espacios de reflexión y acción social relacionados con la elaboración de saberes sobre el tema en cuestión para que les sea útil socialmente y facilite su implementación en la resolución de problemas. Desde esta perspectiva, el investigador en comunicación debe asumir el compromiso moral e ideológico con las luchas sociales con el fin de desarrollar técnicas y estrategias de participación ciudadana para facilitar la indagación de problemáticas específicas en forma colectiva, en virtud de la recuperación crítica de la historia cultural, en términos de diálogos y debates entre los saberes académicos interdisciplinario, en articulación con las experiencias populares.

Bajo este contexto, desde el trabajo de campo -mencionado en la introducción-, asumimos la labor de investigación en vistas de indagar la incidencia pública de las organizaciones sociales (OS), a fin de reconocer, sistematizar y reflexionar con los actores comprometidos, sobre sus acciones en el espacio público para la incidencia política. Para ello, comenzamos a entablar un diálogo con los representantes de InterRedes, una organización social que surgió hace varios años como un espacio de encuentro e intercambio que nuclea a redes de medios populares y comunitarios del país. En el primer encuentro, explicitamos nuestro rol como investigadores en la construcción y reconstitución de los modos de participación en el espacio público y, en tal sentido, del modo de asumir la comunicación estratégica, en términos de comprender las capacidades y habilidades organizacionales para acompañar procesos reflexivos y facilitar acciones que faciliten la transformación de sus realidades coyunturales. Luego, nos dedicamos a explicitar la perspectiva conceptual y metodológica:

- Dar visibilidad a un tema: entendiendo que el primer nivel de incidencia se da cuando la organización percibe o reconoce la existencia de un derecho vulnerado que requiere la atención pública y en consecuencia se moviliza e inicia acciones para instalarlo en la agenda social. 
- Sensibilizar sobre un tema a otros actores clave: el segundo nivel de incidencia es posible cuando la organización promueve el debate y moviliza a otros actores a partir de la conciencia de que los cambios requieren del trabajo conjunto y del apoyo multi-actoral.

- Instalar el tema en la agenda pública: un tercer nivel de incidencia resulta cuando la organización logra que el tema cobre relevancia como prioritario y urgente para la actualidad de la comunidad barrio, municipio, provincia, país o región dependiendo del recorte de territorio que interese-, tanto en los medios de comunicación, en la producción de bienes culturales, en las redes sociales y ámbitos de debate político. Este nivel también implica la sensibilización de actores políticos partidarios y/o gubernamentales clave, a fin de que asuman el tema como parte de su agenda de gestión.

- Participar en la definición de políticas públicas. Una organización se encuentra en el cuarto nivel de incidencia cuando es reconocida y convocada como un actor legítimo para ser parte en alguna o varias etapas del diseño de una política pública, pudiendo además actuar luego como auditor capaz de evaluar su gestión y resultados

A medida que se iban procurando las reuniones fuimos comprobando que en relación a la organización existía la necesidad de diagramar las acciones, en función de considerar los niveles de articulación con los actores claves, los modos de producción de información y conocimiento, las formas de comunicación con la comunidad y el tipo de involucramiento con las políticas públicas. Para ello, se elaboró una serie de interrogante:

\section{Articulación con actores clave}

a) ¿Cuál es el tipo de relación con los actores clave favorables a los objetivos?

b) ¿Cómo es ese vínculo? ¿Con qué grado de frecuencia se encuentran?

c) ¿Se desarrollan estrategias de comunicación para vincularse con los actores clave?

d) ¿Se llevan adelante proyectos conjuntos con otros?

e) ¿Se esta integrado a alguna red?

2.- Construcción de información de y sobre las organizaciones

a) ¿Se cuenta con la información específica sobre la realidad de la comunidad de referencia?

b) ¿Existe una actualización de la información con especialistas y/o fuentes específicas en el tema?

c) ¿Se tiene información sobre el impacto y modalidad de gestión de las políticas públicas vinculadas al tema?

d) ¿Qué acciones de apoyo se realizan para que se profundice la formación en relación a la temática de interés?

3.- Diagnóstico comunicacional y vinculación con la comunidad

a) ¿Se conoce a la población implicada o afectada por la temática que aborda la organización?

b) ¿Se desarrollan instancias de participación con la comunidad para pensar y discutir en conjunto sobre el tema?

c) ¿La población afectada sobre el tema reconoce a la organización?

d) ¿Cuál es la relación con los medios de comunicación locales, regionales y nacionales?

e) ¿Se cuenta con capacidad técnica y/o profesional para desarrollar materiales y estrategias de comunicación?

f) ¿Se apela a recursos de comunicación adecuados para compartir las propuestas con la comunidad?

4. Involucramiento con políticas públicas y legislación 
a) ¿Cómo se involucran con las políticas públicas -municipales, provinciales y nacionales-, vinculadas con el tema de interés?

b) ¿Se conoce a los funcionarios con capacidad de decisión involucrados?

c) ¿La organización es reconocida como referente del tema por parte de los actores gubernamentales?

d) ¿Se cuenta con capacidad técnica para analizar, evaluar y generar propuestas en las políticas públicas?

e) ¿Son convocados por los actores gubernamentales para participar en la definición, implementación y/o evaluación de políticas públicas vinculadas a la temática?

Estos indicadores fueron surgiendo, a partir de los diálogos y debates producidos durante el trascurso de los encuentros. Es así que la información y conocimiento se generó mediante la práctica colectiva, la cual lleva implícitamente a la necesidad de exponer los condicionantes materiales de la organización y las tensiones que existen al interior del grupo para el conocer/hacer en clave de incidencia. Las mismas parten de la obtención de datos y del estudio de las significaciones y sentidos que circulan, en vinculación con las teorías científicas porque consideramos que ni el conocimiento académico ni el popular, aislados, contribuyen a la construcción de saberes sobre lo social que devengan en instrumentos cognitivo para su transformación.

Este modelo de interrelación, en términos de Landry, Amara y Lamari (2001), presta a la identificación de lo que los actores sociales piensan, sienten o teorizan sobre las preguntas emergidas que serán los problemas de investigación. Dicha interacción viabiliza el compartir y discutir la información con las personas o grupos involucrados, conduciendo a descubrir nuevas categorías e hipótesis de trabajo, promoviendo la experimentación con metodologías que combinan técnicas de investigación con la praxis social, dando cuenta de la totalidad compleja y contradictoria de la realidad para desenvolver acciones que siembren el trabajo cognitivo del grupo para la construcción de conocimientos. En tal sentido, las decisiones de un diseño proyectual deben ser compartidas con los grupos involucrados admitiendo que los saberes producidos tienen que enlazarse con los cotidianos y científico. Esto permite la participación activa de la población involucrada en el proceso de objetivación de la realidad y de la relación dialéctica entre teoría y práctica, con un doble objetivo: el de producir conocimiento colectivo y de promover cambios en y de las condiciones que afectan la vida cotidiana de dichos sectores.

$\mathrm{Al}$ concebir a la investigación y la participación como instancias de un mismo proceso de producción de conocimiento, el investigador en comunicación se desenvuelve como experto y como animador socio/ cultural. Tal es así que las acciones que proponga deberán permitir la exposición de las tensiones y disputas por los sentidos que están en juego en la realidad cotidiana del grupo en cuestión para transformarlos en objeto de análisis que llevaran a la determinación de fines y medios para el cambio social.

\section{ConCLUSIón}

Este artículo ha pretendido hacer visible las propias prácticas de investigación en comunicación admitiendo que el conocimiento producido tiene que enlazar los saberes populares con el científico mediante la participación activa de todos los involucrados en dicho proceso.

Definiendo a la participación como la proyección de acciones que permiten la irrupción del encuentro sociocultural de las personas configurando "las tramas de relaciones comunicacionales conformando la densidad de la cultura" (Uranga y Thompson, 2016, p 38), este enfoque abre el debate hacia el replanteamiento epistemológico, político, y por tanto metodológico de la investigación en comunicación debido a que el mismo permite abordar la producción de conocimiento desde las prácticas sociales, en términos procesuales para asumir críticamente la realidad y elaborar estrategias (https://www.monografias .com/trabajos 11/henrym/henrym.shtml) concretas y realizables, en función de la planificación de acciones renovadas en la que intervenga la comunidad para la incidencia política. 


\section{REFERENCIAS}

Aguerre, N. (2017) Sobre el concepto de ciudadanía desde una mirada comunicacional. Revista Question. Recuperado de: https://perio.unlp.edu.ar/ojs/index.php/question/article/view/4286.

Freire, P. (2005). Pedagogía del oprimido. Ciudad Autónoma de Buenos Aires, Argentina: Siglo XXI.

Landry, R; Amara, N; Lamari, M. (2001) Utilization of social science research knowledge in Canada [Utilización del conocimiento de investigación en ciencias sociales en Canadá]. Research Policy, (30), 333-349.

Uranga, W. (2014). Comunicación para la incidencia política. Propuesta de matriz para analizar capacidades y habilidades comunicacionales de las organizaciones sociales en la acción política. [Entrada de blog]. Recuperado de http://www.wuranga.com.ar/images/propios/23_Comunicacin_para_la_incidencia_poltica_ W_Uranga_ALAIC\%20_2014.pdf.

Uranga, W.; Thompson, H. (Coord.) (2016). La incidencia como camino para la construcción de ciudadanía. Buenos Aires, Argentina: Patria Grande.

Weiss, C. (1979). The many meaning of research utilization [Los múltiples significados de la utilización de la investigación]. Public AdministrationReview, 39 (5), 426-431.

\section{Notas}

i Enmarcado en el Programa de Incentivos a la Investigación del Ministerio de Educación de la Nación, el proyecto es dirigido por el profesor Washington Uranga (periodo 2015-2019). Ver blog del proyecto: http://investigacionwu.blo gspot.com/ 